$\mathrm{LA}--10150$

DE34 016976

\title{
The Force Balance Limiting Current for Charged Particle Beams
}

\author{
Abraham Kadish
}

\section{DISCLAIMER}

This report was prepared as an account of work sponsored by an agency of the United States Government. Neither the United States Government nor any agency thereof, nor any of their employees, makes any warranty, express or implied, or assumes any legal liability or responsibility for the accuracy, completeness, or usefulness of any information, apparatus, product, or process disclosed, or represents that its use would not infringe privately owned rights. Reference herein to any specific commercial product, process, or service by trade name, trademark, manufacturer, or otherwise does not necessarily constitute or imply its endorsement, recommendation, or favoring by the United States Government or any agency thereof. The views and opinions of authors expressed herein do not necessarily state or reflect those of the United States Government or any agency thereof.

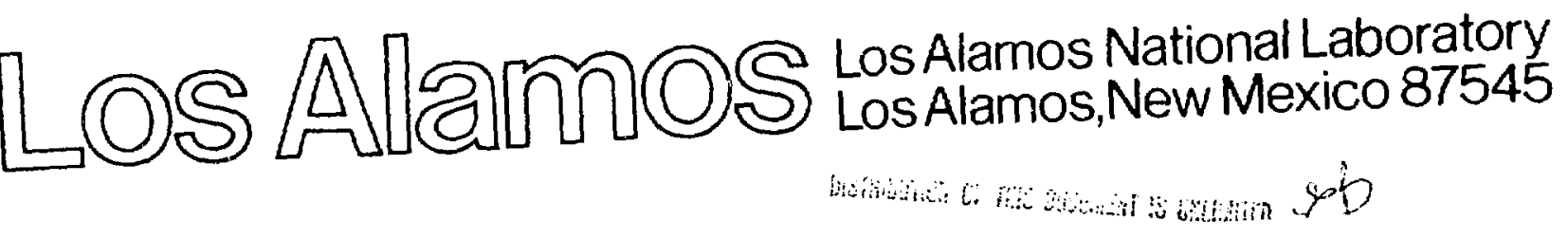




\title{
THE FORCE BALANCE LIMITING CURRENT FOR CHARGED PARTICLE BEAMS
}

\author{
Abraham Kadish
}

\begin{abstract}
A liniting current for cold, relativistic, charged particle beams is derived. Arbitrary rotation and axial flow profiles are allowed. Only force balance is required. A comparison with the usual Brillouin condition reveals a significantly more restrictive scaling for ambitious beam parameters.
\end{abstract}

\section{INTRODUCTION}

Limiting currents for steady state charged particle beams are central to the design of microwave generating devices, accelerators, and propagating plasmoids. Three basic limitations exi: These correspond to ipacecharge limiting currents, in limiting currents, and force balance limiting curr... * Space-charge limiting currents are associated with restrictions on the energy density that may not be exceeded by a beam in the presence of a conducting boundary. An infinite guide field is assumed in the derivation of these criteria so that the beam particle paths are determined. 'Alfvên limiting currents are found by assuming current density profiles for a beam in a charge neutralizing medium and examining test particle orbits in the configuration. The current channel is determined by the propagating test particles. ${ }^{2}$ Force balance limitations are obtained by solving the steady state momentum balance equation with self-consistent fields assuming a finite externally applied guide field. A commonly used criterion obtained from this calculation is the Brillouin condition. ${ }^{3.4}$ Roughly speaking, the Brillouin condition assumes a rigidly rotating beam and predicts that the maximum steady state current scales as the square of both the beam radius and applied guide field.

In this paper, we derive a new bound on the force balance limiting current without assuming rigid beam rotation. We find a more restrictive bound on the current than that given by the Brillouin condition. Thus, for large values of beam radius and guide field, we derive an approximately linear, rather than quadratic, scaling. At small values, exact agreement is obtained. "Equilibria" which satisfy the Brillouin condition but violate the one derived here will fail to satisfy the positivity of $\gamma^{-2}=1-(\mathrm{v} / \mathrm{c})^{2}$.
The next section gives a brief review of the derivation of the Brillouin condition and introduces notation. In Sec. III, we derive the new scaling for arbitrary beam rotation. The final section compares the new scaling with the Brillouin condition.

\section{THE BRILLOUIN CONDITION}

We assume an azimuthally symmetric cold relativistic plasma with charge $q$ and rest mass $m$ per particle. The axial coordinate is taken to be ignorable. The electric field in the beam is assumed to be entirely due to the beam charge, and is therefore radial. The plasma flow and associated self-magnetic field are restricted to lie in surfaces of constant radius, r. An externally supplied guide field in the axial direction, $B_{0}$, is assumed. Employing mks units, force balance is given by

$0=\frac{\beta_{\partial}^{2} \gamma}{r}+\frac{q}{m c}\left[\frac{E_{r}}{c}+\left(\beta_{\theta} B_{z}-\beta_{z} B_{\theta}\right)\right]$.

In Eq. (1), $\gamma$ is the usual relativistic mass factor. Subscripts of $r, \theta$ and $z$ refer to vector components in the radial, azimuthal and axial directions, respectively. The speed of light is $c$. The vector $\vec{\beta}$ is the flow velocity normalized by $c$. The vectors $\vec{E}$ and $\vec{B}$ are the electric and magnetic fields, respectively.

The Brillouin condition for the existence of a solution to Eq. (1) is derived by assuming a rigid rotation of the beam. Thus,

$\beta_{\theta} c=r \Omega ; \frac{d \Omega}{d r}=0$. 
Employing Gauss's Law of electrostatics and the steady state form of Ampere's Law, we find (in mks units)

$$
\begin{aligned}
& E_{r}=\frac{1}{r} \frac{N(r) q}{2 \pi \varepsilon_{0}} ; N(r)=2 \pi \int_{0}^{r} r^{\prime} n\left(r^{\prime}\right) d r^{\prime} \\
& B_{\theta}=\frac{\mu_{0}}{r} \frac{I_{z}}{2 \pi} ; l_{z}(r)=2 \pi \int_{0}^{r} r^{\prime} q c \beta_{z} n\left(r^{\prime}\right) d r^{\prime} \\
& B_{z}=B_{0}+\mu_{0} \int_{r}^{R} q c \beta_{\theta} n\left(r^{\prime}\right) d r^{\prime} .
\end{aligned}
$$

In Eq. (3), $B_{0}$ is the applied guide field and $R$ is the outer beam radius. $\mu_{0}=4 \pi \times 10^{-7}$ and $\varepsilon_{0} \mu_{0}=c^{-2}$.

Employing Eqs. (2) and (3) in Eq. (1) yields a quadratic in $\Omega$ after making the approximation

$$
\gamma \equiv\left(1-\beta^{2}\right) \cong\left(1-\beta_{z}^{2}\right)^{-1 / 2} \cong \gamma_{z} \text {. }
$$

The necessary and sufficient condition for the quadratic to have real solutions (i.e., real $\Omega$ ) is that

$\left|\mathrm{I}_{\mathrm{z}}\right| \leqq \frac{2 \pi}{\mu_{\mathrm{o}}}\left|\frac{\mathrm{mc}}{\mathrm{q}}\right| \frac{1}{4}\left(\frac{\mathrm{qB}_{\mathrm{o}}}{\mathrm{m}} \frac{\mathrm{R}}{\mathrm{c}}\right)^{2} \Gamma<\beta_{\mathrm{z}}>$.

In Eq. (5) we have introduced the quantities $\left\langle\beta_{z}\right\rangle$ and $\Gamma$ defined by

$$
\begin{aligned}
& I_{\mathrm{z}}(\mathrm{R})=\mathrm{qcN}(\mathrm{R})<\beta_{\mathrm{z}}> \\
& \Gamma=\frac{\sqrt{1-\beta_{\mathrm{z}}^{2}(\mathrm{R})}}{1-\beta_{\mathrm{z}}(\mathrm{R})<\beta_{\mathrm{z}}>} .
\end{aligned}
$$

Thus, $\left\langle\beta_{z}\right\rangle$ is the average value of $\beta_{z}$ and $\Gamma$ reduces to $\gamma_{z}$ for uniform axial flow. It is easy to show that $\Gamma \leqq\left(1-\left\langle\beta_{\mathrm{z}}\right\rangle^{2}\right)^{-1 / 2}$. The inequality given in Eq. (5) is the Brillouin condition.

We note that the usual form of the Brillouin condition is not that given by $\mathrm{Eq}$. (5), but rather as a ratio of frequencies. Using the relations given in Eq. (3), we derive

$$
\frac{\left\langle\omega_{p}^{2}\right\rangle}{\omega_{c}^{2}} \equiv \frac{\frac{N}{\left(\pi R^{2}\right)} \frac{q^{2}}{\varepsilon_{0} m}}{q^{2} B_{0}^{2} / m^{2}}<\frac{\Gamma}{2} .
$$

We also note that once the relationship given by Eq. (4) is employed, there is nothing in the derivation of the Brillouin condition to guarantee that solutions for the rotational flow will be compatible with real $\gamma$.

\section{THE FORCE BALANCE LIMITING CUR- RENT}

In this section, we derive a new bound on the limiting current for axially symmetric relativistic charged particle beams. We assume that the axial coordinate is ignorable. However, no a priori assumption is made regarding the rotational flow profile. Moreover, no approximation of $\gamma$ is made to simplify the mathematics.

We begin with Eq. (1), requiring radial force balance, and introduce the quantity $\mu(r)$, defined below, in place of $\beta_{\theta}(r)$.

$\beta_{0}=-\mu \sqrt{1-\beta_{2}^{2}} \operatorname{sgn}\left(q B_{z}\right)$.

We observed that since $1-\beta^{2}$ must be non-negative, $\mu^{2}$ $<1$. Moreover, Eqs. (1) and (3) show that $q \beta_{\theta} B_{z}<0$. That is. the Lorenz force due to rotational flow must be radially inward for equilibrium. Therefore, $\mu$ is positive. Consequently, in the analysis which follows. we only consider positive values of $\mu$ which are less than unity.

Employing Eq. (8) in Eqs. (1) and (3), one finds, after some aigebra, that at each value of $r$, we must have

$\left|\frac{\mathrm{qB}_{\mathrm{z}}}{\mathrm{m}} \frac{\mathrm{r}}{\mathrm{c}}\right|-\frac{\mu}{\sqrt{1-\mu^{2}}}=\frac{1}{\mu}\left[\frac{\mu_{0}}{2 \pi}\left(\left\langle\beta_{\mathrm{z}}>\Gamma_{2}\right)^{-1}\right] \frac{\mathrm{q} i}{\mathrm{mc}}\right.$.

In Eq. (9), $\left\langle\beta_{z}\right\rangle$ is the average value of $\beta_{z}$ in the radial region between zero and $r . \Gamma_{z}$ is of the same form as that given in Eq. (6). However, $\beta_{z}$ is evaluated at $r$, and the value of $\left\langle\beta_{z}\right\rangle$ is the one described above. The quantity I is the axial current flowing in the radial region between zero and $r$.

The graphs of the right- and left-hand sides of Eq. (9) are shown in Figs. 1 and 2 . In drawing these graphs. we have assumed that $\Gamma_{z}>0$. If there is a fractional charge neutralization, $f$, of the beam which carries no current, then the numerator in the defining equation for $\Gamma_{z}$ will have an additional term given by $-f$. If this term is of a magnitude sufficient to make $\Gamma_{z}$ non-positive, then the graph of the right-hand side of Eq. (9) must be reflected across the $\mu$-axis, and it is then easily seen that the two graphs will intersect at precisely one value of $\mu$. Thus, a charge neutralized beam will always have a steady state. If, however, $\Gamma_{z}>0$, we see that the two graphs only intersect if $|q I|$ is not too large. Owing to the opposing convexity of the two graphs, there will be two local rotational flows if $|\mathrm{I}|$ is less than the critical current, and none (no steady state) above the critical current. At the 


$$
\underline{\underline{E}}
$$




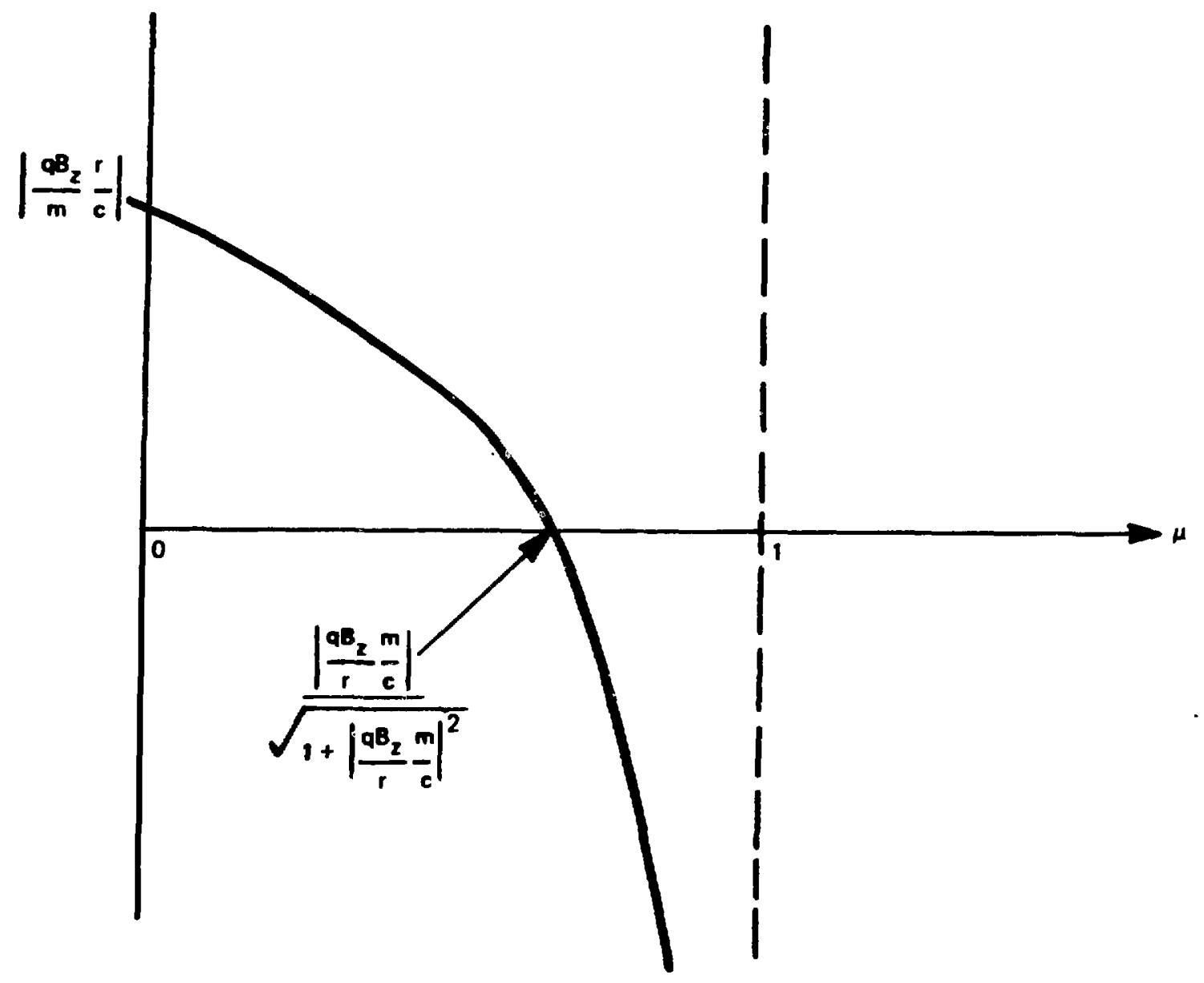

Fig. 2. The graph of the left-hand side of Eq. (9). The convexity is negative. The vertical line through $\mu=1$ is an asymptote of the graph. The graph crosses the $\mu$-axis at

$$
\mu=\frac{r}{c}\left|\frac{q B_{2}}{m}\right|\left[1+\left(\frac{r}{c} \frac{q B_{2}}{m}\right)\right]^{-1 / 2} .
$$

\section{COMPARISON OF LIMITING CURRENTS}

This section is devoted to a discussion of properties of the bound given in Eq. (12) and a comparison of the bound with the Brillouin condition as given by Eq. (5).

We begin by noting that since $\mu$ is always between zero and unity, the coefficient $\mu\left(2-\mu^{2}\right)^{-1}$ in Eq. (12) is also bounded by zero and unity. Using Eq. (9) and (10) at $r=R$, we may eliminate $I$ to obtain $\mu$ as a function of the dimensionless parameter

$$
\rho \equiv\left|\begin{array}{ll}
\frac{q B_{0}}{m} & \frac{R}{c}
\end{array}\right|
$$

Thus,

$\rho=\frac{\mu}{\sqrt{1-\mu^{2}}}\left(\frac{2-\mu^{2}}{1-\mu^{2}}\right)$. 
From Eq. (4), we see that for small values of $\rho, \mu$ is asymptotically given by $\rho / 2$. For $\rho \gg 1, \mu$ is approximately equal to unity. Thus,

$\frac{\mu}{2-\mu^{2}} \sim\left\{\begin{array}{c}\frac{1}{4}\left|\frac{\mathrm{qB}_{0}}{\mathrm{~m}} \frac{\mathrm{R}}{\mathrm{c}}\right| \\ 1\end{array} \quad \begin{array}{l}\mathrm{RB}_{\mathrm{o}} \rightarrow 0 \\ \mathrm{RB}_{\mathrm{o}} \rightarrow \infty\end{array}\right.$.

Note that the result for $R B_{o} \rightarrow 0$ when substituted in Eq. (12) gives the Brillouin condition. This is not surprising since the rotational flow of a small bore beam is asymptotically rigid rotation. However, at the opposite end of the parameter regime. large $\rho$, the Brillouin condition yields an unsatisfactory guideline since it provides a quadratic bound on the current in $\rho$, while the new bound shows that a linear bound is all one can hope for.

In fact, it is easy to show that throughout the range of $\rho$. the new bound is always more restrictive than that given by the Brillouin condition. To see this, we consider the function $D(\rho)$ given by

$D(\rho) \equiv \frac{1}{4} \rho-\left(\frac{\mu}{2-\mu^{2}}\right)$

We already know that $D(0)=0$. We shall now show, using Eq. (14), that $D^{\prime}(\rho)>0$ for $\rho>0$. Differentiating Eq. (14) with respect to $\rho$ yields

$\frac{d \mu}{d \rho}=\frac{\left(1-\mu^{2}\right)^{5 / 2}}{2+\mu^{2}}>0$.

Using Eq. (17) and differentiating Eq. (16), we obtain

$D^{\prime}(\rho)=\frac{1}{4}-\frac{\left(1-\mu^{2}\right)^{5 / 2}}{\left(2-\mu^{2}\right)^{2}}$

$D^{\prime \prime}(\rho)=+\frac{\mathrm{d} \mu}{\mathrm{d} \rho} \frac{\left(3-\mu^{2}\right)}{\left(2-\mu^{2}\right)^{3}} \mu\left(1-\mu^{2}\right)^{3 / 2}$.

We note that $D^{\prime}(0)=0$ while $D^{\prime \prime}(\rho) \geqq 0$. From Eq. (18), we conclude that the Brillouin bound on the current always exceeds the newly derived bound, and the excess is a monotonic function of the parameter
An evaluation of the function $\mu\left(2-\mu^{2}\right)^{-1}$ and a comparison of the new bound with the Brillouin condition is provided in Table I. For a species with charge $Z_{q_{e}}$ and rest mass $\mathrm{m}, \rho$ is given by $0.587 \mathrm{~B}(\mathrm{kG}) \mathrm{R}(\mathrm{cm}) \mathrm{Z} \mathrm{m}_{\mathrm{c}} / \mathrm{m}$. Thus, if we are dealing with electrons, a beam with a 1/2-inch radius in a guide field of $10 \mathrm{kG}$ corresponds to a $\mu$ of about 0.85 . For this beam, the Brillouin condition overestimates the maximum: current by a factor of about 2.8. On the other hand. for the same geometry and guide field, if ions are employed, the bounds are in excellent agreement owing to the small value of the electron-toion mass ratio. Thus, with a $10 \mathrm{kG}$ guide field an ion beam radius in excess of a meter and a quarter would have to be considered before the two bounds differed by more than $2 \%$.

TABLE I. Comparison of Factors in Brillouin and Force Balance Limiting Expressions. Selected pairs of $\mu$ and $\rho$ are displayed in the first two colur ns of the table. The third and fourth columns are corresponding factors in the expressions for the force balance and Brillouin limiting currents. The last column is the ratio of the Brillouin limiting current and the force balance critical current. (i.e., the ratio of the numbers in columns four and three of this table).

\begin{tabular}{lrlll}
\hline & $\frac{\mathrm{qB}}{\mathrm{m}} \frac{\mathrm{R}}{\mathrm{c}}$ & $\frac{\mu}{2-\mu^{2}}$ & $\frac{1}{4} \frac{\mathrm{qB}}{\mathrm{m}} \frac{\mathrm{R}}{\mathrm{c}}$ & $\frac{\mathrm{I}_{\mathrm{Br}}}{\mathrm{I}_{\text {crit }}}$ \\
\hline 0 & 0.000 & 0.0000 & 0.0000 & 1.00 \\
0.1 & 0.202 & 0.0502 & 0.0505 & 1.01 \\
0.2 & 0.417 & 0.102 & 0.104 & 1.02 \\
0.3 & 0.660 & 0.157 & 0.165 & 1.05 \\
0.4 & 0.956 & 0.217 & 0.239 & 1.10 \\
0.5 & 1.347 & 0.286 & 0.337 & 1.18 \\
0.6 & 1.922 & 0.366 & 0.480 & 1.31 \\
0.7 & 2.902 & 0.464 & 0.726 & 1.56 \\
0.8 & 5.037 & 0.588 & 1.259 & 2.14 \\
0.85 & 7.428 & 0.665 & 1.857 & 2.79 \\
0.9 & 12.932 & 0.756 & 3.233 & 4.25 \\
0.92 & 17.630 & 0.798 & 4.408 & 5.52 \\
0.94 & 26.425 & 0.842 & 6.606 & 7.85 \\
0.95 & 34.247 & 0.866 & 8.561 & 9.89 \\
0.96 & 47.160 & 0.890 & 11.790 & 13.25 \\
0.97 & 71.504 & 0.916 & 17.876 & 19.52 \\
0.98 & 129.285 & 0.943 & 32.321 & 34.27 \\
0.99 & 359.677 & 0.971 & 89.419 & 92.09 \\
\hline \hline
\end{tabular}
$\left|\frac{\mathrm{qB}_{0}}{\mathrm{~m}} \frac{\mathrm{R}}{\mathrm{c}}\right|$. 


\section{ACKNOWLEDGMENT}

I would like to thank Bernadette Archuleta for formatting and editing this paper.

\section{REFERENCES}

1. B. N. Brejzman and D. D. Ryutov, Nucl. Fusion 14, 873 (1974).

2. H. Alfvén. Phỵs. Rev. 55, 425 (1939).

3. R. C. Davidson, Theory of Nonneutral Plasmas (W.

A. Benjamin. Inc., Reading. Mass., 1974).

4. R. B. Miller, Intense Charged Particle Beams (Plenum Press. New York, 1982).

5. P. Diament, Phys. Rev. Lett. 37, 168 (1976).

6. A. Kadish, Phys. Fluids 24, 1358 (1981).

מU.S. GOVERNMENT PRINTING OFFICE: 1984-776.026/4140 